\title{
Soliton Solutions for Quasilinear Schrödinger Equations
}

\author{
Junheng Qu \\ Information Science and Department of Mathematics, Science School of Foshan University, Foshan, Guangdong 528000, China
}

Correspondence should be addressed to Junheng Qu; ququ0315@hotmail.com

Received 28 June 2013; Accepted 6 September 2013

Academic Editor: Abdul Hamid Kara

Copyright (C) 2013 Junheng Qu. This is an open access article distributed under the Creative Commons Attribution License, which permits unrestricted use, distribution, and reproduction in any medium, provided the original work is properly cited.

By using a change of variables, we get new equations, whose respective associated functionals are well defined in $H^{1}\left(\mathbb{R}^{N}\right)$ and satisfy the geometric hypotheses of the mountain pass theorem. Using this fact, we obtain a nontrivial solution.

\section{Introduction}

We study the existence of solutions for the following quasilinear Schrödinger equations:

$$
\begin{array}{r}
-\Delta u+V(x) u-\left[\Delta\left(1+u^{2}\right)^{\alpha / 2}\right] \frac{\alpha u}{2\left(1+u^{2}\right)^{(2-\alpha) / 2}}= \\
x \in u^{q}+u^{p}, \\
x \in \mathbb{R}^{N},
\end{array}
$$

where $V \in C\left(\mathbb{R}^{N}, \mathbb{R}^{+}\right)$is bounded and periodic in each variable of $x_{i}, 1 \leq i \leq N, N \geq 3, T(\alpha)<q+1<p+1<$ $\alpha 2^{*}:=2 \alpha N /(N-2), \alpha \geq 1$, and here

$$
T(\alpha):= \begin{cases}2 \alpha, & \alpha_{0} \leq \alpha, \\ 2 \alpha_{0}, & 1<\alpha<\alpha_{0}, \\ 12-4 \sqrt{6}, & \alpha=1,\end{cases}
$$

where $\alpha_{0}$ is defined in Lemma 2. These equations are related to existence of standing wave solutions for quasilinear Schrödinger equations of the form

$$
\begin{array}{r}
i z_{t}=-\Delta z+W(x) z-h\left(|z|^{2}\right) z-\Delta g\left(|z|^{2}\right) g^{\prime}\left(|z|^{2}\right) z, \\
x \in \mathbb{R}^{N},
\end{array}
$$

where $W$ is a given potential and $g$ and $h$ are real functions. Quasilinear equations such as (3) have been accepted as models of several physical phenomena corresponding to various types of $g$. The case of $g(s)=s^{\alpha}$ was used for the superfluid film equation in plasma physics [1]. Besides, (3) also appears in plasma physics and fluid mechanics [2], in dissipative quantum mechanics [3], and in the theory of Heisenberg ferromagnetism and magnons $[4,5]$. See also $[6,7]$ for more physical backgrounds. Equations (3) with $\alpha=1$ have been studied extensively recently; see $[8,9]$. When $g(s)=(1+s)^{\alpha / 2}$, then (3) turn into our equations (1) with $h(s)=s^{q}+s^{p}$. In particular if we let $\alpha=1$, that is, $g(s)=(1+s)^{1 / 2}$, (3) models the self-channeling of a high-power ultrashort laser in matter [10]. In this case, few results are known. In [11], the authors proved global existence and uniqueness of small solutions in transverse space dimensions 2 and 3 and local existence without any smallness condition in transverse space dimension 1. In [12], the authors proved the existence of nontrivial solution. When $\alpha>1$, although we do not know the physical background of (3), in a mathematical sense, we give the proof of the existence of nontrivial solution.

For (1), the main difficulty is that the energy functional associated to (1) is not well defined in $H^{1}\left(\mathbb{R}^{N}\right)$. To overcome this difficulty, enlightened by $[8,9]$, we give a new change of variables. Then we reduce the quasilinear problem (1) to a semilinear one, which we will prove has a nontrivial solutions.

Our main result is the following.

Theorem 1. Assume that $\alpha \geq 1$ and $T(\alpha)<q+1<p+1<$ $\alpha 2^{*}$. Then (1) has a nontrivial solution.

In this paper, $C$ denotes positive (possibly different) constant, $L^{p}\left(\mathbb{R}^{N}\right)$ denotes the usual Lebesgue space with norm 
$|u|_{p}=\left(\int_{\mathbb{R}^{N}}|u|^{p} d x\right)^{1 / p}, 1 \leq p<\infty$, and $H^{1}\left(\mathbb{R}^{N}\right)$ denotes the Sobolev space with norm $\|u\|=\left(\int_{\mathbb{R}^{N}}\left(|\nabla u|^{2}+V(x) u^{2}\right) d x\right)^{1 / 2}$.

\section{The Change of Variables}

We note that the solutions of (1) are the critical points of the following functional:

$$
\begin{aligned}
I(u)= & \frac{1}{2} \int_{\mathbb{R}^{N}}\left[1+\frac{\alpha^{2} u^{2}}{2\left(1+u^{2}\right)^{2-\alpha}}\right]|\nabla u|^{2} d x \\
& +\frac{1}{2} \int_{\mathbb{R}^{N}} V(x) u^{2} d x \\
& -\frac{1}{q+1} \int_{\mathbb{R}^{N}} u^{q+1} d x-\frac{1}{p+1} \int_{\mathbb{R}^{N}} u^{p+1} d x .
\end{aligned}
$$

Since the functional $I(u)$ may not be well defined in the usual Sobolev spaces $H^{1}\left(\mathbb{R}^{N}\right)$, we make a change of variables as

$$
v=G(u)=\int_{0}^{u} g(t) d t,
$$

where $g(t)=\sqrt{1+\alpha^{2} t^{2} / 2\left(1+t^{2}\right)^{2-\alpha}}$. Since $g(t)$ is monotonous with $|t|$, the inverse function $G^{-1}(t)$ of $G(t)$ exists. Then after the change of variables, $I(u)$ can be written by

$$
\begin{aligned}
J(v)= & \frac{1}{2} \int_{\mathbb{R}^{N}}|\nabla v|^{2} d x+\frac{1}{2} \int_{\mathbb{R}^{N}} V(x)\left|G^{-1}(v)\right|^{2} d x \\
& -\frac{1}{q+1} \int_{\mathbb{R}^{N}}\left|G^{-1}(v)\right|^{q+1} d x \\
& -\frac{1}{p+1} \int_{\mathbb{R}^{N}}\left|G^{-1}(v)\right|^{p+1} d x .
\end{aligned}
$$

By Lemma 2 listed below, we have $\lim _{t \rightarrow 0} G^{-1}(t) / t=1$ and $\lim _{t \rightarrow \infty}\left|G^{-1}(t)\right|^{\alpha} / t=\sqrt{2}(\alpha>1)$ or $\sqrt{2 / 3}(\alpha=1)$, so $J(v)$ is well defined in $H^{1}\left(\mathbb{R}^{N}\right)$ and $J(v) \in C^{1}$.

If $u$ is a nontrivial solution of (1), then for all $\phi \in C_{0}^{\infty}\left(\mathbb{R}^{N}\right)$ it should satisfy

$$
\begin{gathered}
\int_{\mathbb{R}^{N}}\left[g^{2}(u) \nabla u \nabla \phi+g(u) g^{\prime}(u)|\nabla u|^{2} \phi+V(x) u \phi\right. \\
\left.-u^{q} \phi-u^{p} \phi\right] d x=0 .
\end{gathered}
$$

We show that (7) is equivalent to

$$
\begin{aligned}
J^{\prime}(v) \psi=\int_{\mathbb{R}^{N}}[ & \nabla \nu \psi+V(x) \frac{G^{-1}(v)}{g\left(G^{-1}(v)\right)} \psi \\
& \left.-\frac{\left|G^{-1}(v)\right|^{q}}{g\left(G^{-1}(v)\right)} \psi-\frac{\left|G^{-1}(v)\right|^{p}}{g\left(G^{-1}(v)\right)} \psi\right] d x
\end{aligned}
$$

$=0, \quad \forall \psi \in C_{0}^{\infty}\left(\mathbb{R}^{N}\right)$.
Indeed, if we choose $\phi=(1 / g(u)) \psi$ in (7), then we get (8). On the other hand, since $u=G^{-1}(v)$, if we let $\psi=g(u) \phi$ in (8), we get (7). Therefore, in order to find the nontrivial solutions of (1), it suffices to study the existence of the nontrivial solutions of the following equations:

$$
\begin{array}{r}
-\Delta v=-V(x) \frac{G^{-1}(v)}{g\left(G^{-1}(v)\right)}+\frac{\left|G^{-1}(v)\right|^{q}}{g\left(G^{-1}(v)\right)}+\frac{\left|G^{-1}(v)\right|^{p}}{g\left(G^{-1}(v)\right)}, \\
x \in \mathbb{R}^{N} .
\end{array}
$$

Before we close this section, we give some properties of the change of variables.

Lemma 2. For all $t>0$, one has the following:

(1) $\lim _{t \rightarrow 0}\left(G^{-1}(t) / t\right)=1$,

(2) (i) if $\alpha>1$ then $\lim _{t \rightarrow \infty}\left(\left|G^{-1}(t)\right|^{\alpha} / t\right)=\sqrt{2}$, and (ii) if $\alpha=1$ then $\lim _{t \rightarrow \infty}\left(\left|G^{-1}(t)\right| / t\right)=\sqrt{2 / 3}$,

(3) $\left|G^{-1}(t)\right| \leq t$,

(4) (i) if $\alpha_{0} \leq \alpha$ then $\operatorname{tg}^{\prime}(t) / g(t) \leq \alpha-1$, (ii) if $1<\alpha \leq$ $\alpha_{0}$ then $\operatorname{tg}^{\prime}(t) / g(t) \leq \alpha_{0}-1$, and (iii) if $\alpha=1$ then $\operatorname{tg}^{\prime}(t) / g(t) \leq 5-2 \sqrt{6}$, where $\alpha_{0} \approx 1.36$ is a real root of the equation $\alpha^{3}-4 \alpha^{2}+8 \alpha-6=0$.

Proof. (1) We easily get $\lim _{t \rightarrow 0}\left(G^{-1}(t) / t\right)=\left.\left(G^{-1}(t)\right)^{\prime}\right|_{t=0}=$ $1 / g\left(G^{-1}(0)\right)=1$.

For (2) if $\alpha>1$, since $g(t)=\sqrt{1+\alpha^{2} t^{2} / 2\left(1+t^{2}\right)^{2-\alpha}}=$ $\sqrt{1+\left(\alpha^{2} t^{2} / 2\left(1+t^{2}\right)\right)\left(1+t^{2}\right)^{\alpha-1}}$, so $g(t) \sim \sqrt{\left(\alpha^{2} / 2\right) t^{2(\alpha-1)}}=$ $(\alpha / \sqrt{2}) t^{\alpha-1}$ as $t \rightarrow \infty$, then $G(t)=\int_{0}^{t} g(s) d s \sim(1 / \sqrt{2}) t^{\alpha}$ as $t \rightarrow \infty$. Since $G^{-1}(t)$ is the inverse of $G(t)$, so $G^{-1}(t) \sim$ $(\sqrt{2} t)^{1 / \alpha}$ as $t \rightarrow \infty$, thus we have $\lim _{t \rightarrow \infty}\left(\left|G^{-1}(t)\right|^{\alpha} / t\right)=\sqrt{2}$.

When $\alpha=1$, the result is obvious since $g(t)$ is an increasing bounded function.

For (3), since $\left[G^{-1}(t)-(1 / g(0)) t\right]^{\prime}=1 / g\left(G^{-1}(t)\right)-1 /$ $g(0) \leq 0$, so $G^{-1}(t) \leq(1 / g(0)) t=t$, which proves (3).

Now we prove $(4)$, since $(t / g(t)) g^{\prime}(t)=t^{2} /$ $2\left(1+t^{2}\right)^{2} g^{2}(t)=t^{2} /\left(2+5 t^{2}+3 t^{4}\right)=1 /\left(2 / t^{2}+5+3 t^{2}\right) \leq$ $5-2 \sqrt{6}$, which is (iii). To prove (i), that is,

$$
\alpha^{2}(2-\alpha) t^{2} \leq 2(\alpha-1)\left(1+t^{2}\right)^{3-\alpha},
$$

we set $j(t)=2(\alpha-1)\left(1+t^{2}\right)^{3-\alpha}-\alpha^{2}(2-\alpha) t^{2}$, so $j^{\prime}(t)=$ $2 t\left[2(\alpha-1)(3-\alpha)\left(1+t^{2}\right)^{2-\alpha}-\alpha^{2}(2-\alpha)\right]:=2 t k_{\alpha}(t)$, where $k_{\alpha}(t)=2(\alpha-1)(3-\alpha)\left(1+t^{2}\right)^{2-\alpha}-\alpha^{2}(2-\alpha)$. Then $k_{\alpha}^{\prime}(t)=$ $4(\alpha-1)(3-\alpha)(2-\alpha) t\left(1+t^{2}\right)^{1-\alpha}$. If $\alpha \leq 2$ or $\alpha \geq 3$, we get $k_{\alpha}^{\prime}(t) \geq 0$, so $k_{\alpha}(t) \geq k_{\alpha}(0)$. We notice that $k_{\alpha}(0)=\alpha^{3}-4 \alpha^{2}+$ $8 \alpha-6$ and $k_{\alpha}(0)$ is an increasing function with respect to $\alpha$. By Cardano's formula for cubic equations, we know that $k_{\alpha}(0)$ has one real root and two complex roots. If we set $\alpha_{0} \approx 1.36$ to be the real root of $k_{\alpha}(0)$, then $k_{\alpha}(t) \geq k_{\alpha}(0) \geq 0$ as $\alpha \geq \alpha_{0}$. 
So $j^{\prime}(t)=2 t k_{\alpha}(t) \geq 0$. That is $j(t)$ is a increasing function, so $j(t) \geq j(0)=2(\alpha-1)>0$ as $\alpha>1$. If $2<\alpha<3$, we get $k_{\alpha}^{\prime}(t)<0$, so $k_{\alpha}(t)$ is a decreasing function, but in this case $\lim _{t \rightarrow \infty} k_{\alpha}(t)=(\alpha-2) \alpha^{2}>0$, so $k_{\alpha}(t) \geq 0$ for all $t>0$. Thus we have the same result as $\alpha \leq 2$ or $\alpha \geq 3$, which proves (i). For (ii), by the definition of $\alpha_{0}$, we have

$$
\frac{\alpha_{0}^{2} t^{2}\left(1+\left(\alpha_{0}-1\right) t^{2}\right)}{2\left(1+t^{2}\right)^{3-\alpha_{0}}+\alpha_{0}^{2} t^{2}\left(1+t^{2}\right)} \leq \alpha_{0}-1,
$$

so

$$
\begin{aligned}
& \alpha_{0}^{2} t^{2}\left(1+\left(\alpha_{0}-1\right) t^{2}\right) \\
& \quad \leq 2\left(\alpha_{0}-1\right)\left(1+t^{2}\right)^{3-\alpha_{0}}+\alpha_{0}^{2}\left(\alpha_{0}-1\right) t^{2}\left(1+t^{2}\right) .
\end{aligned}
$$

We add $\left(\alpha_{0}-1\right)\left(\alpha^{2}-\alpha_{0}^{2}\right) t^{2}\left(1+t^{2}\right)$ to both sides of (12), where $\alpha<\alpha_{0}$. Then

$$
\begin{aligned}
& \alpha_{0}^{2} t^{2}+\alpha_{0}^{2}\left(\alpha_{0}-1\right) t^{4}+\left(\alpha_{0}-1\right)\left(\alpha^{2}-\alpha_{0}^{2}\right)\left(t^{2}+t^{4}\right) \\
& \quad \leq 2\left(\alpha_{0}-1\right)\left(1+t^{2}\right)^{3-\alpha_{0}}+\left(\alpha_{0}-1\right) \alpha^{2} t^{2}\left(1+t^{2}\right), \\
& \therefore\left(\alpha_{0}^{2}+\left(\alpha_{0}-1\right)\left(\alpha^{2}-\alpha_{0}^{2}\right)\right) t^{2}+\left(\alpha_{0}-1\right) \alpha^{2} t^{4} \\
& \quad \leq\left(\alpha_{0}-1\right)\left[2\left(1+t^{2}\right)^{3-\alpha}+\alpha^{2} t^{2}\left(1+t^{2}\right)\right] .
\end{aligned}
$$

We notice that $\alpha_{0}^{2}+\left(\alpha_{0}-1\right)\left(\alpha^{2}-\alpha_{0}^{2}\right) \geq \alpha^{2}$. In fact, $\alpha_{0}^{2}+\alpha_{0} \alpha^{2}-$ $\alpha_{0}^{3}-\alpha^{2}+\alpha_{0}^{2} \geq \alpha^{2} \Leftrightarrow 2\left(\alpha_{0}^{2}-\alpha^{2}\right)+\alpha_{0}\left(\alpha^{2}-\alpha_{0}^{2}\right) \geq 0 \Leftrightarrow$ $\left(\alpha_{0}^{2}-\alpha^{2}\right)\left(2-\alpha_{0}\right)>0$, and the last inequality is obvious. So

$$
\begin{aligned}
\alpha^{2} t^{2}+ & \left(\alpha_{0}-1\right) \alpha^{2} t^{4} \\
& \leq\left(\alpha_{0}-1\right)\left[2\left(1+t^{2}\right)^{3-\alpha}+\alpha^{2} t^{2}\left(1+t^{2}\right)\right] \\
\therefore \alpha^{2} t^{2} & +(\alpha-1) \alpha^{2} t^{4} \\
& \leq\left(\alpha_{0}-1\right)\left[2\left(1+t^{2}\right)^{3-\alpha}+\alpha^{2} t^{2}\left(1+t^{2}\right)\right],
\end{aligned}
$$

which implies that $\operatorname{tg}^{\prime}(t) / g(t) \leq \alpha_{0}-1$.

\section{Mountain Pass Geometry}

In this section, we establish the geometric hypotheses of the mountain pass theorem.

Lemma 3. There exist $\rho_{0}, a_{0}>0$ such that $J(v) \geq a_{0}$ for all $\|v\|=\rho_{0}$.

Proof. Let

$$
\begin{aligned}
Q(x, t):= & -\frac{1}{2} V(x)\left|G^{-1}(t)\right|^{2}+\frac{1}{q+1}\left|G^{-1}(t)\right|^{q+1} \\
& +\frac{1}{p+1}\left|G^{-1}(t)\right|^{p+1} .
\end{aligned}
$$

Then, by Lemma 2 and $p+1<\alpha 2^{*}$, we have

$$
\begin{aligned}
& \lim _{t \rightarrow 0} \frac{Q(x, t)}{t^{2}}=\lim _{t \rightarrow 0}[- \frac{1}{2} V(x)\left(\frac{G^{-1}(t)}{t}\right)^{2} \\
&+\frac{1}{q+1}\left(\frac{G^{-1}(t)}{t}\right)^{2}\left|G^{-1}(t)\right|^{q-1} \\
&\left.+\frac{1}{p+1}\left(\frac{G^{-1}(t)}{t}\right)^{2}\left|G^{-1}(t)\right|^{p-1}\right] \\
&=-\frac{1}{2} V(x),
\end{aligned}
$$$$
\lim _{t \rightarrow \infty} \frac{Q(x, t)}{t^{2^{*}}}=\lim _{t \rightarrow \infty}\left[-\frac{1}{2} V(x)\left(\frac{\left|G^{-1}(t)\right|^{\alpha}}{t}\right)^{2 / \alpha} \frac{1}{t^{2^{*}-2 / \alpha}}\right.
$$$$
+\frac{1}{q+1}\left(\frac{\left|G^{-1}(t)\right|^{\alpha}}{t}\right)^{(q+1) / \alpha} \frac{1}{t^{2^{*}-(q+1) / \alpha}}
$$$$
\left.+\frac{1}{p+1}\left(\frac{\left|G^{-1}(t)\right|^{\alpha}}{t}\right)^{(p+1) / \alpha} \frac{1}{t^{2^{*}-(p+1) / \alpha}}\right]
$$

$=0$.

Thus, for $\epsilon>0$ sufficiently small, there exists a constant $C_{\epsilon}>$ 0 such that

$$
Q(x, t) \leq\left(-\frac{1}{2} V(x)+\epsilon\right) t^{2}+C_{\epsilon}|t|^{2^{*}} .
$$

Then, we have

$$
\begin{aligned}
J(v)= & \frac{1}{2} \int_{\mathbb{R}^{N}}|\nabla v|^{2} d x+\frac{1}{2} \int_{\mathbb{R}^{N}} V(x)\left|G^{-1}(v)\right|^{2} d x \\
& -\frac{1}{q+1} \int_{\mathbb{R}^{N}}\left|G^{-1}(v)\right|^{q+1} d x-\frac{1}{p+1} \\
& \times \int_{\mathbb{R}^{N}}\left|G^{-1}(v)\right|^{p+1} d x \\
\geq & \frac{1}{2} \int_{\mathbb{R}^{N}}|\nabla v|^{2} d x+\frac{1}{2} \int_{\mathbb{R}^{N}} V(x) v^{2} d x-\epsilon \\
& \times \int_{\mathbb{R}^{N}} v^{2} d x-C_{\epsilon} \int_{\mathbb{R}^{N}} v^{2^{*}} d x \\
\geq & C\|v\|^{2}-C\|v\|^{2^{*}} .
\end{aligned}
$$

Thus, by choosing $\rho_{0}$ small, we get the result when $\|v\|=\rho_{0}$.

Lemma 4. There exists $v \in H^{1}\left(\mathbb{R}^{N}\right)$ such that $J(v)<0$.

Proof. Given $\phi \in C_{0}^{\infty}\left(\mathbb{R}^{N},[0,1]\right)$ with supp $\phi:=\bar{B}_{1}$, we will prove that $J(s \phi) \rightarrow-\infty$ as $s \rightarrow \infty$, which will prove 
the result if we take $v=s \phi$ with $s$ large enough. By the proof of Lemma 2, we have $G^{-1}(t) \geq C t^{1 / \alpha}$ as $t \geq 1$, so

$$
\begin{aligned}
J(s \phi) \leq & \frac{1}{2} s^{2} \int_{\mathbb{R}^{N}}|\nabla \phi|^{2} d x+\frac{1}{2} s^{2} \\
& \times \int_{\mathbb{R}^{N}} V(x) \phi^{2} d x-s^{(p+1) / \alpha} \\
& \times \int_{\{|s \phi| \geq 1\}} \phi^{(p+1) / \alpha} d x \longrightarrow-\infty,
\end{aligned}
$$

as $s \rightarrow \infty$. Thus, we get the result.

\section{Existence}

In consequence of Lemmas 3 and 4 of the AmbrosettiRabinowitz mountain pass Theorem [13], see also [14-16], for the constant

$$
c=\inf _{\gamma \in \Gamma_{t \in[0,1]}} \sup _{t} J(\gamma(t))>0,
$$

where $\Gamma=\left\{\gamma \in C\left([0,1], H^{1}\left(\mathbb{R}^{N}\right)\right): \gamma(0)=0, \gamma(1) \neq 0\right.$, $J(\gamma(1))<0\}$, and there exists a Palais-Smale seq-uence at level $c$; that is, $J\left(v_{n}\right) \rightarrow c$ and $J^{\prime}\left(v_{n}\right) \rightarrow 0$ as $n \rightarrow \infty$.

Lemma 5. The Palais-Smale sequence $\left\{v_{n}\right\}$ for $J$ is bounded in $H^{1}\left(\mathbb{R}^{N}\right)$.

Proof. Since $\left\{v_{n}\right\} \subset H^{1}\left(\mathbb{R}^{N}\right)$ satisfies

$$
\begin{aligned}
J\left(v_{n}\right)= & \frac{1}{2} \int_{\mathbb{R}^{N}}\left|\nabla v_{n}\right|^{2} d x+\frac{1}{2} \int_{\mathbb{R}^{N}} V(x)\left|G^{-1}\left(v_{n}\right)\right|^{2} d x \\
& -\frac{1}{p+1} \int_{\mathbb{R}^{N}}\left|G^{-1}\left(v_{n}\right)\right|^{p+1} d x \\
& -\frac{1}{q+1} \int_{\mathbb{R}^{N}}\left|G^{-1}\left(v_{n}\right)\right|^{q+1} d x=c+o(1)
\end{aligned}
$$

and for any $\psi \in C_{0}^{\infty}\left(\mathbb{R}^{N}\right)$,

$$
\begin{aligned}
J^{\prime}\left(v_{n}\right) \psi=\int_{\mathbb{R}^{N}}\left[\nabla v_{n} \nabla \psi+V(x) \frac{G^{-1}\left(v_{n}\right)}{g\left(G^{-1}\left(v_{n}\right)\right)} \psi\right. \\
\left.\quad-\frac{\left|G^{-1}\left(v_{n}\right)\right|^{q}}{g\left(G^{-1}\left(v_{n}\right)\right)} \psi-\frac{\left|G^{-1}\left(v_{n}\right)\right|^{p}}{g\left(G^{-1}\left(v_{n}\right)\right)} \psi\right] d x \\
=o(1)\|\psi\| .
\end{aligned}
$$

Now, we consider the function $G^{-1}\left(v_{n}\right) g\left(G^{-1}\left(v_{n}\right)\right)$. Note by Lemma 2 that

$$
\begin{aligned}
\left|\nabla\left(G^{-1}\left(v_{n}\right) g\left(G^{-1}\left(v_{n}\right)\right)\right)\right| \\
\quad=\left[1+\frac{G^{-1}\left(v_{n}\right)}{g\left(G^{-1}\left(v_{n}\right)\right)} g^{\prime}\left(G^{-1}\left(v_{n}\right)\right)\right]\left|\nabla v_{n}\right| \\
\quad \leq \frac{1}{2} T(\alpha)\left|\nabla v_{n}\right| .
\end{aligned}
$$

Combining Lemma 2, we have $G^{-1}\left(v_{n}\right) g\left(G^{-1}\left(v_{n}\right)\right) \in H^{1}\left(\mathbb{R}^{N}\right)$. Thus, since $C_{0}^{\infty}\left(\mathbb{R}^{N}\right)$ is dense in $H^{1}\left(\mathbb{R}^{N}\right)$, by choosing $\psi=$ $G^{-1}\left(v_{n}\right) g\left(G^{-1}\left(v_{n}\right)\right)$ in (23), we deduce that

$$
\begin{gathered}
o(1)\left\|v_{n}\right\|=J^{\prime}\left(v_{n}\right) G^{-1}\left(v_{n}\right) g\left(G^{-1}\left(v_{n}\right)\right) \\
=\int_{\mathbb{R}^{N}}\left[\left(1+\frac{G^{-1}\left(v_{n}\right)}{g\left(G^{-1}\left(v_{n}\right)\right)} g^{\prime}\left(G^{-1}\left(v_{n}\right)\right)\right)\left|\nabla v_{n}\right|^{2}\right. \\
+V(x)\left|G^{-1}\left(v_{n}\right)\right|^{2}-\left|G^{-1}\left(v_{n}\right)\right|^{q+1} \\
\left.-\left|G^{-1}\left(v_{n}\right)\right|^{p+1}\right] d x \\
\leq \int_{\mathbb{R}^{N}}\left[\frac{1}{2} T(\alpha)\left|\nabla v_{n}\right|^{2}+V(x)\left|G^{-1}\left(v_{n}\right)\right|^{2}\right. \\
\left.-\left|G^{-1}\left(v_{n}\right)\right|^{q+1}-\left|G^{-1}\left(v_{n}\right)\right|^{p+1}\right] d x .
\end{gathered}
$$

Therefore, by (22) and (25), we have

$$
\begin{aligned}
(q+1) & J\left(v_{n}\right)-J^{\prime}\left(v_{n}\right) G^{-1}\left(v_{n}\right) g\left(G^{-1}\left(v_{n}\right)\right) \\
\geq & \left(\frac{q+1}{2}-\frac{1}{2} T(\alpha)\right) \\
& \times \int_{\mathbb{R}^{N}}\left|\nabla v_{n}\right|^{2} d x+\frac{q-1}{2} \int_{\mathbb{R}^{N}} V(x)\left|G^{-1}\left(v_{n}\right)\right|^{2} d x \\
& +\left(1-\frac{q+1}{p+1}\right) \int_{\mathbb{R}^{N}}\left|G^{-1}\left(v_{n}\right)\right|^{p+1} d x \\
\geq & \left(\frac{q+1}{2}-\frac{1}{2} T(\alpha)\right) \int_{\mathbb{R}^{N}}\left|\nabla v_{n}\right|^{2} d x+\frac{q-1}{2} \\
& \times \int_{\mathbb{R}^{N}} V(x)\left|G^{-1}\left(v_{n}\right)\right|^{2} d x .
\end{aligned}
$$

Combining (22) and (26), we get $\int_{\mathbb{R}^{N}}\left|G^{-1}\left(v_{n}\right)\right|^{p+1} d x$ is bounded. To verify that $\left\{v_{n}\right\}$ is bounded in $H^{1}\left(\mathbb{R}^{N}\right)$ we start splitting

$$
\begin{aligned}
\int_{\mathbb{R}^{N}} V(x) v_{n}^{2} d x= & \int_{\left\{x:\left|v_{n}(x)\right|>1\right\}} V(x) v_{n}^{2} d x \\
& +\int_{\left\{x:\left|v_{n}(x)\right| \leq 1\right\}} V(x) v_{n}^{2} d x .
\end{aligned}
$$

By the proof of Lemma 2, we have $G^{-1}(t) \geq C t^{1 / \alpha}$, for all $t>1$ and $G(1)=\int_{0}^{1} g(t) d t \geq \int_{0}^{1} d t=1$. Therefore

$$
\begin{aligned}
& \int_{\left\{x:\left|v_{n}(x)\right|>1\right\}} V(x) v_{n}^{2} d x \\
& \quad \leq C \int_{\left\{x:\left|v_{n}(x)\right|>1\right\}}\left|G^{-1}\left(v_{n}\right)\right|^{2 \alpha} d x \\
& \quad \leq C \int_{\left\{x:\left|v_{n}(x)\right|>1\right\}}\left|G^{-1}\left(v_{n}\right)\right|^{p+1} d x \leq C .
\end{aligned}
$$


Since $g(t)$ is increasing and $G(t)=\int_{0}^{t} g(s) d s \leq g(t) t$, we have

$$
\begin{aligned}
& \int_{\left\{x:\left|v_{n}(x)\right| \leq 1\right\}} V(x)\left|G^{-1}\left(v_{n}\right)\right|^{2} d x \\
& \quad \geq \frac{1}{g^{2}\left(G^{-1}(1)\right)} \int_{\left\{x:\left|v_{n}(x)\right| \leq 1\right\}} V(x) v_{n}^{2} d x .
\end{aligned}
$$

Hence $\left\{v_{n}\right\}$ is bounded in $H^{1}\left(\mathbb{R}^{N}\right)$, and this proves Lemma 5 .

Now we give the completion of the proof of Theorem 1.

Proof. First, we will prove that $J^{\prime}(v)=0$. That is, $v$ is a weak solution of (9). To prove this, it suffices to show that

$$
\begin{array}{r}
J^{\prime}(v) \psi=\int_{\mathbb{R}^{N}}\left[\nabla v \nabla \psi+V(x) \frac{G^{-1}(v)}{g\left(G^{-1}(v)\right)} \psi\right. \\
\left.-\frac{\left|G^{-1}(v)\right|^{q}}{g\left(G^{-1}(v)\right)} \psi-\frac{\left|G^{-1}(v)\right|^{p}}{g\left(G^{-1}(v)\right)} \psi\right] d x=0, \\
\forall \psi \in C_{0}^{\infty}\left(\mathbb{R}^{N}\right) .
\end{array}
$$

From Lemma $5,\left\{v_{n}\right\}$ is a bounded Palais-Smale sequence, and there exists $v \in H^{1}\left(\mathbb{R}^{N}\right)$ such that $v_{n} \rightarrow v$ weakly in $H^{1}\left(\mathbb{R}^{N}\right)$. By the Lebesgue dominated theorem, we have

$$
\begin{aligned}
& J^{\prime}\left(v_{n}\right) \psi-J^{\prime}(v) \psi \\
& =\int_{\mathbb{R}^{N}}\left(\nabla v_{n}-\nabla v\right) \nabla \psi d x \\
& \quad+\int_{\mathbb{R}^{N}} V(x)\left[\frac{G^{-1}\left(v_{n}\right)}{g\left(G^{-1}\left(v_{n}\right)\right)}-\frac{G^{-1}(v)}{g\left(G^{-1}(v)\right)}\right] \psi d x \\
& \quad-\int_{\mathbb{R}^{N}}\left[\frac{\left|G^{-1}\left(v_{n}\right)\right|^{q}}{g\left(G^{-1}\left(v_{n}\right)\right)}-\frac{\left|G^{-1}(v)\right|^{q}}{g\left(G^{-1}(v)\right)}\right] \psi d x \\
& \quad-\int_{\mathbb{R}^{N}}\left[\frac{\left|G^{-1}\left(v_{n}\right)\right|^{p}}{g\left(G^{-1}\left(v_{n}\right)\right)}-\frac{\left|G^{-1}(v)\right|^{p}}{g\left(G^{-1}(v)\right)}\right] \psi d x \rightarrow 0 .
\end{aligned}
$$

Hence, $J^{\prime}(v)=0$. That is, $v$ is a weak solution of (1).

Next, in order to complete the proof of Theorem 1, we must show that $v$ is nontrivial. By contradiction, we assume $v=0$. To prove this, we claim that, for all $R>0$,

$$
\lim _{n \rightarrow \infty} \sup _{y \in \mathbb{R}^{N}} \int_{B_{R}(y)} v_{n}^{2} d x=0
$$

cannot occur. Suppose by contradiction that (32) occurs; that is, $\left\{v_{n}\right\}$ vanishes. Then by the Lions compactness lemma [16], $v_{n} \rightarrow 0$ in $L^{r}\left(\mathbb{R}^{N}\right)$ for any $r \in\left(2,2^{*}\right)$. By the proof of Lemma 2 , we get $G^{-1}(t):(\sqrt{2} t)^{1 / \alpha}$ as $t \rightarrow \infty$, so there exists a suitable constant $C$ such that $G^{-1}(t) \leq C t^{1 / \alpha}$. In addition, since $G(t) \leq g(t) t$, we have

$$
\begin{aligned}
& \lim _{n \rightarrow \infty} \int_{\mathbb{R}^{N}} \frac{\left|G^{-1}\left(v_{n}\right)\right|^{p}}{g\left(G^{-1}\left(v_{n}\right)\right)} v_{n} d x \\
& \leq \lim _{n \rightarrow \infty} \int_{\mathbb{R}^{N}}\left|G^{-1}\left(v_{n}\right)\right|^{p+1} d x \\
& \leq \lim _{n \rightarrow \infty} C \int_{\mathbb{R}^{N}} v_{n}^{(p+1) / \alpha} d x=0, \\
& \lim _{n \rightarrow \infty} \int_{\mathbb{R}^{N}}\left|G^{-1}\left(v_{n}\right)\right|^{q+1} d x \\
& \quad=\lim _{n \rightarrow \infty} \int_{\mathbb{R}^{N}}\left|G^{-1}\left(v_{n}\right)\right|^{p+1} d x=0,
\end{aligned}
$$

and $\lim _{n \rightarrow \infty} \int_{\mathbb{R}^{N}}\left(\left|G^{-1}\left(v_{n}\right)\right|^{q} / g\left(G^{-1}\left(v_{n}\right)\right)\right) v_{n} d x=0$ is obvious since $q<p$, which implies that

$$
\begin{aligned}
0= & \lim _{n \rightarrow \infty} J^{\prime}\left(v_{n}\right) v_{n} \\
= & \lim _{n \rightarrow \infty} \int_{\mathbb{R}^{N}}\left[\left|\nabla v_{n}\right|^{2}+V(x) \frac{G^{-1}\left(v_{n}\right)}{g\left(G^{-1}\left(v_{n}\right)\right)} v_{n}\right. \\
& \left.\quad-\frac{\left|G^{-1}\left(v_{n}\right)\right|^{q}}{g\left(G^{-1}\left(v_{n}\right)\right)} v_{n}-\frac{\left|G^{-1}\left(v_{n}\right)\right|^{p}}{g\left(G^{-1}\left(v_{n}\right)\right)} v_{n}\right] d x \\
= & \lim _{n \rightarrow \infty} \int_{\mathbb{R}^{N}}\left[\left|\nabla v_{n}\right|^{2}+V(x) \frac{G^{-1}\left(v_{n}\right)}{g\left(G^{-1}\left(v_{n}\right)\right)} v_{n}\right] d x .
\end{aligned}
$$

Then,

$$
\begin{gathered}
\lim _{n \rightarrow \infty} \int_{\mathbb{R}^{N}}\left|\nabla v_{n}\right|^{2} d x=0, \\
\lim _{n \rightarrow \infty} \int_{\mathbb{R}^{N}} V(x) \frac{G^{-1}\left(v_{n}\right)}{g\left(G^{-1}\left(v_{n}\right)\right)} v_{n} d x=0 .
\end{gathered}
$$

On the other hand, by (25), we have

$$
\lim _{n \rightarrow \infty} \int_{\mathbb{R}^{N}} V(x)\left|G^{-1}\left(v_{n}\right)\right|^{2} d x=0 .
$$

Combining (35) and (37), we get a contradiction since $J\left(v_{n}\right) \rightarrow c>0$. Thus, $\left\{v_{n}\right\}$ does not vanish and there exist $k, R>0$ and $\left\{y_{n}\right\} \subset \mathbb{R}^{N}$ such that

$$
\lim _{n \rightarrow \infty} \int_{B_{R}\left(y_{n}\right)} v_{n}^{2} d x \geq k>0 .
$$

Define $\widetilde{v}_{n}(x)=v_{n}\left(x+y_{n}\right)$. We may assume that the components of $\left\{y_{n}\right\}$ are integer multiples of the periods of $V(x)$. Since $\left\{v_{n}\right\}$ is a Palais-Smale sequence for $J$ and $V(x)$ is periodic in $x_{i}, 1 \leq i \leq N,\left\{\widetilde{v}_{n}\right\}$ is also a Palais-Smale sequence for $J$ with $J^{\prime}(\widetilde{v})=0$ if $\widetilde{v}_{n} \rightarrow \widetilde{v}$ in $H^{1}\left(\mathbb{R}^{N}\right)$. Since $\left\{\widetilde{v}_{n}\right\}$ does not vanish, we have that $\widetilde{v} \neq 0$ is a nontrivial solution of (9). 


\section{Acknowledgment}

The research is supported by NSF of China (11201154).

\section{References}

[1] S. Kurihara, "Large-amplitude quasi-solitons in superfluid films," Journal of the Physical Society of Japan, vol. 50, no. 10, pp. 3262-3267, 1981.

[2] A. G. Litvak and A. M. Sergeev, "One dimensional collapse of plasma waves," JETP Letters, vol. 27, pp. 517-520, 1978.

[3] R. W. Hasse, "A general method for the solution of nonlinear soliton and kink Schrödinger equations," Zeitschrift für Physik $B$, vol. 37, no. 1, pp. 83-87, 1980.

[4] A. M. Kosevich, B. A. Ivanov, and A. S. Kovalev, "Magnetic solitons," Physics Report, vol. 194, no. 3-4, pp. 117-238, 1990.

[5] G. R. W. Quispel and H. W. Capel, "Equation of motion for the Heisenberg spin chain," Physica A, vol. 110, no. 1-2, pp. 41-80, 1982.

[6] B. Hartmann and W. Zakzewski, "Electrons on hexagonal lattices and applications to nanotubes," Physical Review, vol. 68, pp. 1-9, 2003.

[7] L. Brizhik, A. Eremko, B. Piette, and W. J. Zakrzewski, "Static solutions of a $D$-dimensional modified nonlinear Schrödinger equation," Nonlinearity, vol. 16, no. 4, pp. 1481-1497, 2003.

[8] J.-Q. Liu, Y.-Q. Wang, and Z.-Q. Wang, "Soliton solutions for quasilinear Schrödinger equations. II," Journal of Differential Equations, vol. 187, no. 2, pp. 473-493, 2003.

[9] M. Colin and L. Jeanjean, "Solutions for a quasilinear Schrödinger equation: a dual approach," Nonlinear Analysis: Theory, Methods \& Applications, vol. 56, no. 2, pp. 213-226, 2004.

[10] E. W. Laedke, K. H. Spatschek, and L. Stenflo, "Evolution theorem for a class of perturbed envelope soliton solutions," Journal of Mathematical Physics, vol. 24, no. 12, pp. 2764-2769, 1983.

[11] A. de Bouard, N. Hayashi, and J.-C. Saut, "Global existence of small solutions to a relativistic nonlinear Schrödinger equation," Communications in Mathematical Physics, vol. 189, no. 1, pp. 73105, 1997.

[12] Y. Shen and Y. Wang, "Soliton solutions for generalized quasilinear Schrödinger equations," Nonlinear Analysis: Theory, Methods \& Applications, vol. 80, pp. 194-201, 2013.

[13] M. Schechter, Linking Methods in Critical Point Theory, Birkhäuser, Boston, Mass, USA, 1999.

[14] A. Floer and A. Weinstein, "Nonspreading wave packets for the cubic Schrödinger equation with a bounded potential," Journal of Functional Analysis, vol. 69, no. 3, pp. 397-408, 1986.

[15] L. Jeanjean and K. Tanaka, "A remark on least energy solutions in $\mathbb{R}^{N}$," Proceedings of the American Mathematical Society, vol. 131, no. 8, pp. 2399-2240, 2003.

[16] P. L. Lions, "The concentration compactness principle in the calculus of variations. The locally compact case-part I and II," Annales de l'Institut Henri Poincaré, vol. 1, pp. 109-145, 1984. 


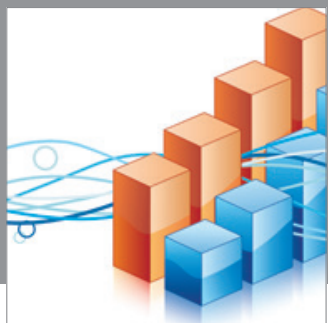

Advances in

Operations Research

mansans

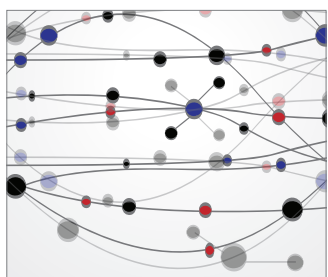

The Scientific World Journal
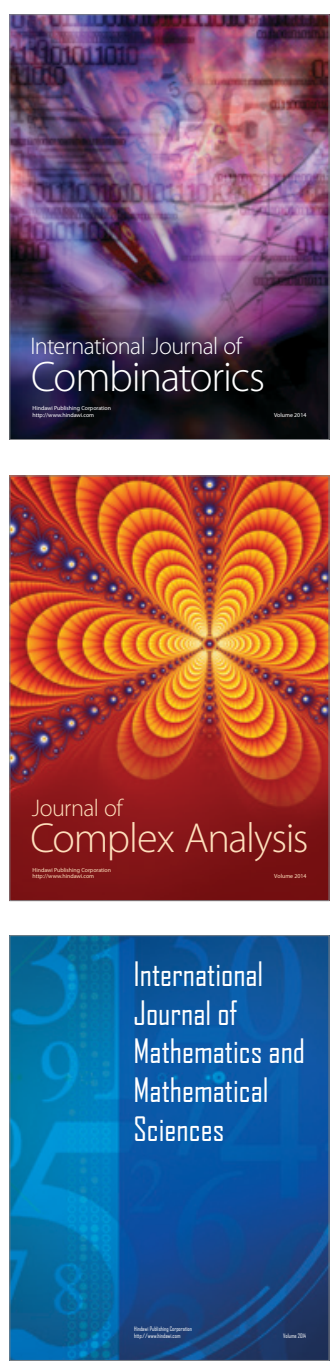
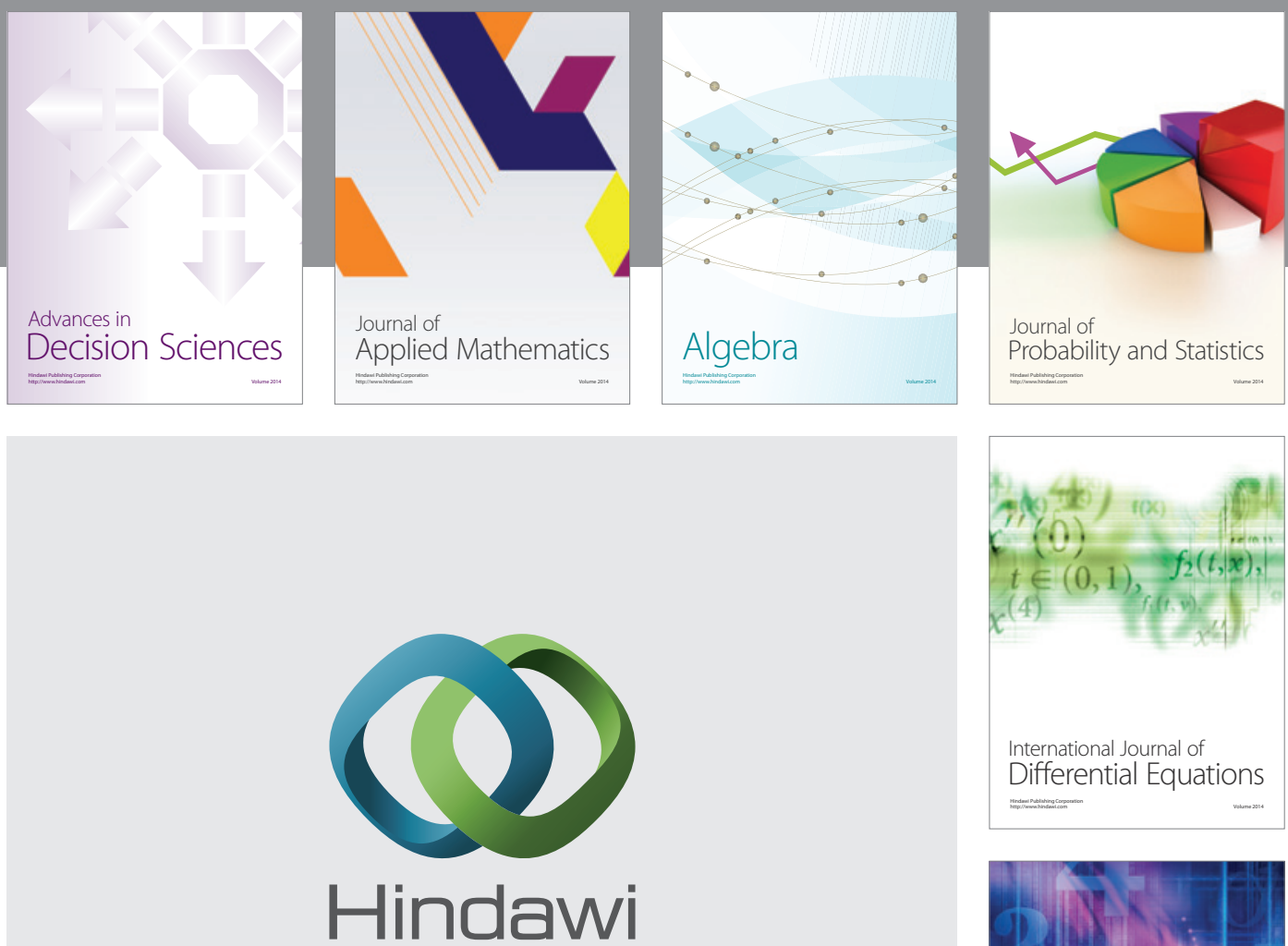

Submit your manuscripts at http://www.hindawi.com
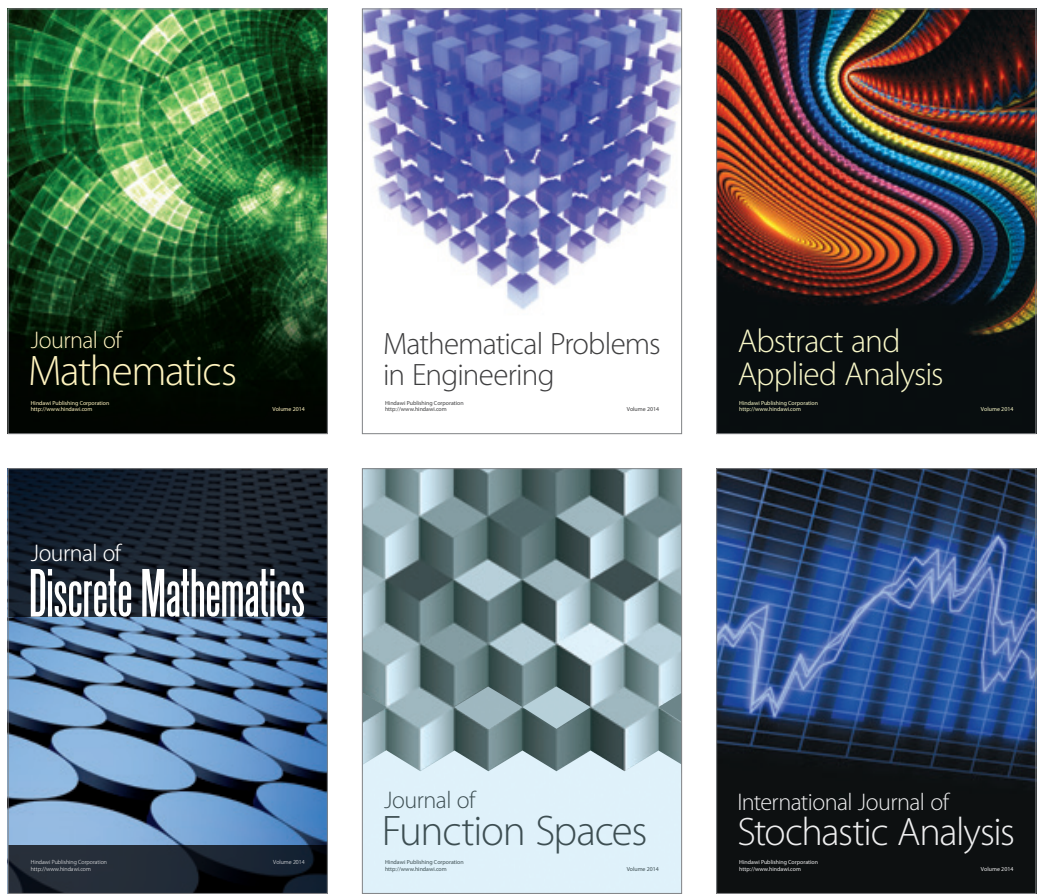

Journal of

Function Spaces

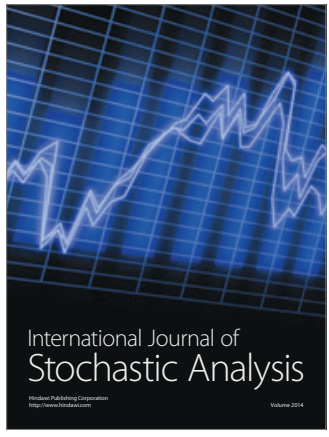

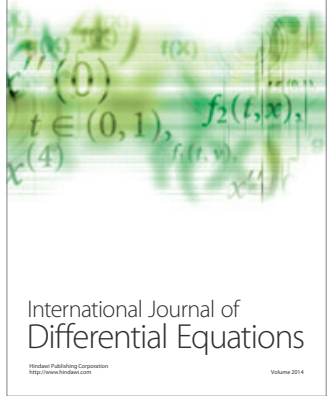
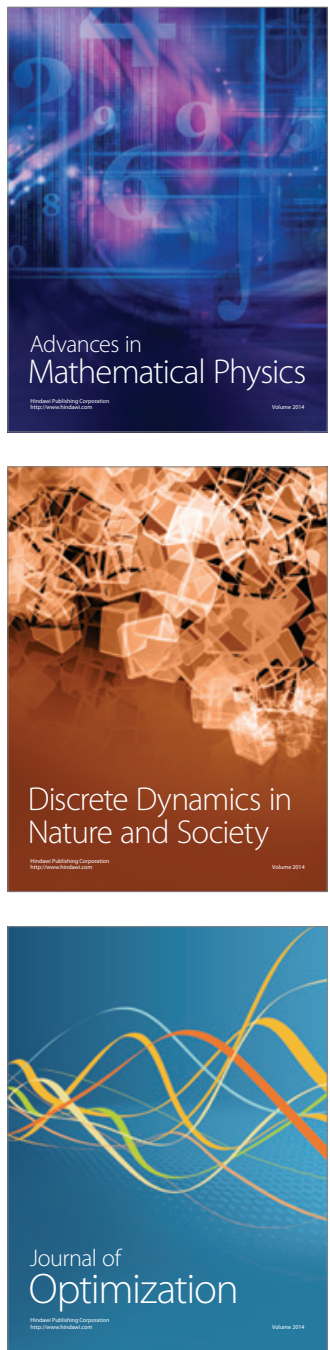